\title{
Assessment of Healthcare Workers' Levels of Preparedness and Awareness Regarding COVID-19 Infection in Low-Resource Settings
}

Muhammed Elhadi, ${ }^{1 \star}$ Ahmed Msherghi, ${ }^{1}$ Mohammed Alkeelani, ${ }^{1}$ Abdulaziz Zorgani, ${ }^{1}$ Ahmed Zaid, ${ }^{1}$ Ali Alsuyihili, ${ }^{1}$ Anis Buzreg, ${ }^{1}$ Hazim Ahmed, ${ }^{1}$ Ahmed Elhadi, ${ }^{1}$ Ala Khaled, ${ }^{1}$ Tariq Boughididah, ${ }^{2}$ Samer Khel, ${ }^{1}$ Mohammed Abdelkabir, ${ }^{3}$ Rawanda Gaffaz, Sumayyah Bahroun, ${ }^{4}$ Ayiman Alhashimi, ${ }^{1,8}$ Marwa Biala, ${ }^{1}$ Siraj Abulmida, ${ }^{1}$ Abdelmunam Elharb, ${ }^{5}$ Mohamed Abukhashem, ${ }^{1}$

Moutaz Elgzairi, ${ }^{2}$ Esra Alghanai, ${ }^{1}$ Taha Khaled, ${ }^{1}$ Esra Boushi, ${ }^{6}$ Najah Ben Saleim, ${ }^{5}$ Hamad Mughrabi, ${ }^{7}$ Nafati Alnafati, ${ }^{1}$ Moaz Alwarfalli, ${ }^{1}$ Amna Elmabrouk, ${ }^{1}$ Sarah Alhaddad, ${ }^{1}$ Farah Madi, ${ }^{1}$ Malack Madi, ${ }^{1}$ Fatima Elkhfeefi, ${ }^{2}$ Mohamed Ismaeil, ${ }^{2}$ Belal Faraag, ${ }^{2}$ Majdi Badi, ${ }^{2}$ Ayman AL-Agile, ${ }^{1}$ Mohamed Eisay, ${ }^{5}$ Jalal Ahmid, ${ }^{1}$ Ola Elmabrouk, ${ }^{1}$ Fatimah Bin Alshiteewi, ${ }^{1}$ Hind Alameen, ${ }^{1}$ Hala Bikhayr, ${ }^{1}$

Tahani Aleiyan, ${ }^{1}$ Bushray Almiqlash, ${ }^{1}$ Malak Subhi, ${ }^{1}$ Mawada Fadel, ${ }^{1}$ Hana Yahya, ${ }^{5}$ Safeya Alkot, ${ }^{5}$ Abdulmueti Alhadi, ${ }^{4}$ Abraar Abdullah, ${ }^{1}$ Abdulrahman Atewa, ${ }^{1}$ and Ala Amshai ${ }^{1}$

${ }^{1}$ Faculty of Medicine, University of Tripoli, Tripoli, Libya; ${ }^{2}$ Faculty of Medicine, University of Benghazi, Benghazi, Libya; ${ }^{3}$ Faculty of Medicine, Sebha University, Sebha, Libya; ${ }^{4}$ Faculty of Medicine, University of Zawia, Az-Zawiyah, Libya; ${ }^{5}$ Faculty of Medicine, University of AL-Mergib, Al Khums, Libya; ${ }^{6}$ Faculty of Medicine, Al-Zintan University, Az-Zintan, Libya; ${ }^{7}$ Faculty of Medicine, Misurata University, Misurata, Libya; ${ }^{8}$ Faculty of Medicine, Al-Jabal Al Gharbi University, Gherian, Libya

\begin{abstract}
COVID-19, caused by the SARS-CoV-2 virus, is spreading rapidly worldwide, with devastating consequences for patients, healthcare workers, health systems, and economies. As it reaches low- and middle-income countries, the pandemic puts healthcare workers at high risk and challenges the abilities of healthcare systems to respond to the crisis. This study measured levels of knowledge and preparedness regarding COVID-19 among physicians and nurses. A cross-sectional survey was conducted among healthcare workers in Libya between February 26 and March 10 , 2020. We obtained 1,572 valid responses of a possible 2,000 (78.6\%) participants from 21 hospitals, of which $65.1 \%$ were from physicians and $34.9 \%$ from nurses. The majority of participants (70\%) used social media as a source of information. A total of $47.3 \%$ of doctors and $54.7 \%$ of nurses received adequate training on how to effectively use personal protective equipment. Low confidence in managing suspected COVID-19 patients was reported by $83.8 \%$ of participants. Furthermore, $43.2 \%$ of healthcare workers were aware of proper hand hygiene techniques. Less than $7 \%$ of participants received training on how to manage COVID-19 cases, whereas $20.6 \%$ of doctors and $26.3 \%$ of nurses felt that they were personally prepared for the outbreak. Awareness and preparedness for the pandemic were low among frontline workers during the study. Therefore, an effective educational training program should be implemented to ensure maintenance of appropriate practices during the COVID-19 pandemic.
\end{abstract}

\section{INTRODUCTION}

In late 2019, a novel COVID-19 was reported to cause severe viral pneumonia in Wuhan, China. It has since spread worldwide, resulting in a pandemic that has now infected more than 1.3 million people, causing more than 80,000 deaths globally. ${ }^{1,2}$ In February 2020, the WHO named the condition COVID-19, which stands for coronavirus disease $2019 .^{3}$

The severity of symptomatic infections ranges from mild to critical. Approximately $80 \%$ of patients have mild symptoms, whereas less than $20 \%$ experience severe symptoms such as dyspnea and shock; respiratory failure occurs in less than $5 \%$ of patients. ${ }^{4-7}$ Elderly patients and/or patients with comorbidities, such as cardiovascular diseases, respiratory diseases, hypertension, diabetes mellitus, and chronic kidney diseases, are at a higher risk for severe illness. They have a higher risk of mortality than younger, or otherwise healthier, individuals. ${ }^{8,9}$ Previous reports have confirmed that hospitalized patients had a mean age of 49-55 years. ${ }^{6,10}$ In an earlier report provided by the United States, regarding COVID-19 patients treated between February 12, 2020 and March 2020, about $67 \%$ of those hospitalized were older than 45 years, which is similar to a prior report from China. ${ }^{11,12}$

Acute respiratory distress syndrome (ARDS) is a significant complication for COVID-19 patients; an estimated $20-41 \%$ of patients develop ARDS following a COVID-19 infection and

*Address correspondence to Muhammed Elhadi, Faculty of Medicine, University of Tripoli, Alfurnaj, University Rd., Tripoli 13275, Libya.

E-mail: muhammed.elhadi.uot@gmail.com require mechanical ventilation. 5,13 This characteristic of the disease can significantly increase the existing burden on healthcare facilities, and it requires extra resources and appropriate management.

COVID-19 poses a higher risk for physicians and nurses who work in critical care, emergency medicine, infectious diseases, and pulmonary medicine departments. Personal protective equipment (PPE), proper handwashing, and hand hygiene are critical in decreasing the transmission and risk of infection of COVID-19 in hospitals. Therefore, adequate training, knowledge, and resources are necessary to prevent hospital-acquired infections due to crosscontamination to other patients who receive care in these departments. ${ }^{14-16}$

As of April 12, 2020, the Libya National Center for Disease Control (NCDC) reported 524 suspected cases of COVID-19, with 25 confirmed diagnoses. ${ }^{17}$ However, these numbers raise a question regarding the local authorities' ability to perform adequate testing for COVID-19, as the Libya NCDC can perform an average of 30 tests per day. Furthermore, given the current civil war, limited financial resources, and a shortage of machines and materials, Libya is more vulnerable to the COVID-19 pandemic. In 2017, the WHO released a report on the healthcare system in Libya, revealing that approximately $20 \%$ of hospitals were shut down. In addition, several major hospitals only operate at $80 \%$ capacity or less. The report states that chronic respiratory disease readiness is less than $29 \%$, whereas the readiness of emergency services is less than $47 \%$ of the target. Furthermore, readiness for emergency 
health services is less than $18 \%$ in terms of training and $40 \%$ for diagnosis abilities. The report indicates that Libya does not have a rapid response team or the ability to diagnose and respond to alerts in an organized manner. ${ }^{18}$ This raises concerns about the local healthcare authorities' preparedness and capacity to provide an adequate response to COVID-19 infection.

Therefore, critical preparedness, readiness, and knowledge regarding COVID-19 are needed for physicians and nurses on the front line. ${ }^{16,19} \mathrm{Few}$ researchers have addressed the overall issues of preparedness of healthcare systems for COVID-19, especially in developing countries, where resources and facilities are limited. Considerable concerns have been raised regarding countries' preparedness for COVID-19 and their ability to maintain control. This study aimed to determine the preparedness and knowledge of Libyan healthcare workers regarding COVID-19, and to develop and validate a measurement tool for estimating healthcare preparedness on a global scale.

\section{METHOD}

Study design. A cross-sectional survey study was conducted in 21 hospitals in Libya between February 26, 2020 and March 10, 2020.

Setting and participants. The study was conducted among physicians and nurses working in the emergency department, intensive care units (ICUs), and respiratory and infectious disease departments, all of whom may expect to encounter COVID-19 patients. The study methodology was explained to the participants, and they were asked to provide consent before participating in the study. Doctors and nurses working in other departments or private clinics were excluded from the study. A total of 2,000 targeted participants were given a paper-based questionnaire at their workplace.

Instruments. The self-administered and anonymous questionnaire was developed by the authors of the study and was validated in a pilot study of 30 participants, who did not participate in the final analysis. Some of the questions were based on a framework similar to that of previous studies on infectious disease outbreaks. ${ }^{20-22}$ The structured questionnaire was validated to address questions about healthcare workers' level of preparedness and knowledge of COVID-19 (Supplemental files I-II).

The first part of the survey was designed to collect data regarding the background characteristics of participants (age, gender, department, years of experience, information sources, and previous experience with outbreaks). The second part comprised seven items intended to evaluate the knowledge and awareness of physicians and nurses regarding COVID-19 infection. The third part comprised 11 items to assess the overall preparedness in terms of managing cases of COVID-19 infection. The questionnaire evaluates information sources, training experience with COVID-19, diagnosis and management of COVID-19 patients, use of PPE, safety precautions, isolation procedures, measures to prevent infection, and reporting procedures. To assess a given participant's knowledge, each correct answer was given a score of 1 , and an incorrect answer was given a zero. Scores were summed for a total score of 7 , ranging from 0 to 7 for knowledge. The preparedness ranges from 0 to 11 . Those who scored $\geq 5$ on the knowledge scale were deemed to have a high level of knowledge, whereas those who scored $\geq 8$ on the preparedness scale had adequate preparedness.

The questionnaire was developed in English for doctors and was translated into Arabic for nurses using a forwardbackward translation by two qualified, independent linguistic translators at the University of Tripoli. Each translator independently performed a forward translation of the original questionnaire. Backward translation from Arabic to English was carried out by another linguistic translator. Three researchers then reviewed the translated versions of the questionnaire for discrepancies. Pilot tests of both versions were carried out, and Cronbach's alpha was used to measure the internal consistency reliability of both versions. Participants were recruited in relative proportion to the estimated number of doctors and nurses working in these departments in Libya. We assumed that $70 \%$ of participants had basic knowledge and preparedness regarding COVID-19, and the estimated sample size was calculated with $80 \%$ power at a $95 \%$ confidence limit, with a design effect equal to 2, and using 20 clusters.

Statistical analysis. Data entry and analyses were performed using SPSS (IBM SPSS Statistics for Windows, version 25.0; IBM Corp., Armonk, NY). Descriptive statistics were used to describe the study variables. Frequency, percentage, and mean scores were used to report the descriptive analysis. The chi-square test was used to assess the association difference between the groups. Statistical significance was considered for $P<0.05$.

Ethical approval. Ethical approval for this study was obtained from the Bioethics Committee at Biotechnology Research Center in Libya [Reference number: BEC-BTRC109.3-2020]. All participants provided consent before participating in the study.

\section{RESULTS}

We obtained 1,572 valid responses out of a possible 2,000 (78.6\%) participants from 21 hospitals in Libya. Among the 1,572 total healthcare personnel, $65.1 \%$ were physicians and $34.9 \%$ were nurses. Table 1 summarizes the characteristics of the study population. Figure 1 provides the distribution of participants based on departments and profession. The mean age of the population $( \pm S D$ ) was $35.5 \pm 7.3$ years for doctors and $27.8 \pm 5.4$ years for nurses, with women making up $64.6 \%$ of all respondents. Approximately half of the participants had low confidence regarding their ability to manage COVID-19 patients. Most participants (70\% of both doctors and nurses) named social media as a source of information for COVID-19, which was statistically significant, whereas fewer than $4 \%$ viewed training courses as such.

Doctors had an average of $7.8 \pm 7.6$ years of healthcare experience, whereas nurses had an average of $5.8 \pm 4.1$ years. All participants worked in general public hospitals: $30.7 \%$ were in ICUs, $58.8 \%$ in the emergency department, $3.2 \%$ in the infectious disease department, and $7.4 \%$ in respiratory departments. A total of $47.3 \%$ of doctors and $54.7 \%$ of nurses did not receive adequate training in the use of PPE. Meanwhile, $16.2 \%$ of doctors and $13.3 \%$ of nurses indicated confidence in their ability to handle suspected COVID-19 patients, versus $83.8 \%$ of doctors and $86.7 \%$ of nurses who did not 
TABLE 1

Baseline characteristics across the study population

\begin{tabular}{|c|c|c|c|}
\hline Characteristic & Doctors $(n=1,024), n(\%)$ & Nurses $(n=548), n(\%)$ & $x^{2}$ \\
\hline Age range (years) & & & $<0.001 \dagger$ \\
\hline$<35$ & $580(56.6)$ & $481(87.8)$ & \\
\hline$\geq 35$ & $444(43.4)$ & $67(12.2)$ & \\
\hline Gender & & & 0.156 \\
\hline Male & $375(36.6)$ & $181(33)$ & \\
\hline Female & 649 (63.4) & $367(67)$ & \\
\hline Department & & & $<0.001 \dagger$ \\
\hline Emergency department & $587(57.3)$ & $337(61.5)$ & \\
\hline Intensive care units & $306(29.9)$ & $176(32.1)$ & \\
\hline Respiratory department & $89(8.7)$ & $8(1.5)$ & \\
\hline Infectious disease department & $42(4.1)$ & $27(4.9)$ & \\
\hline Previous experience of outbreak & & & $>0.05$ \\
\hline SARS & $57(5.6)$ & $32(5.8)$ & \\
\hline MERS & $25(2.4)$ & $17(3.1)$ & \\
\hline Bird flu & $114(11.1)$ & 74 (13.5) & \\
\hline Other & $17(1.7)$ & $7(1.3)$ & \\
\hline Years of experience & & & 0.323 \\
\hline$<5$ & $477(46.6)$ & $241(44)$ & \\
\hline$\geq 5$ & $547(53.4)$ & $307(56)$ & \\
\hline \multicolumn{4}{|l|}{ Sources of information } \\
\hline Media (TV and radio) & $665(65.1)$ & $331(60.8)$ & 0.093 \\
\hline Social media & $715(70.1)$ & $412(75.7)$ & $0.018^{*}$ \\
\hline Training courses & $37(3.6)$ & $13(2.4)$ & 0.185 \\
\hline Discussion with colleagues & $202(19.2)$ & $103(17.3)$ & 0.225 \\
\hline Online courses & 125 (12.3) & $47(8.6)$ & $0.03^{\star}$ \\
\hline $\begin{array}{l}\text { Confidence in management of COVID-19 } \\
\text { patients }\end{array}$ & & & 0.428 \\
\hline No confidence & $510(49.9)$ & $275(50.2)$ & \\
\hline Little confidence & $348(34)$ & $200(36.5)$ & \\
\hline Confidence & $110(10.8)$ & $46(8.4)$ & \\
\hline High confidence & $55(5.4)$ & $27(4.9)$ & \\
\hline
\end{tabular}

* Significant at $P<0.05$.

† Significant at $P<0.001$

indicate confidence in this regard. About $45 \%$ of doctors and $37 \%$ of nurses indicated that they were not prepared to handle cases of infection transmitted at the hospital level.

The measurements of knowledge, awareness, and preparedness are presented in Table 2 . The reliability of the knowledge and preparedness questionnaires was determined using Cronbach's alpha, which revealed scores of 0.72 and 0.68 for the knowledge and preparedness scales, respectively. The results showed that less than half of all participants knew the symptoms of COVID-19 infection (44.4\% of doctors and $31.7 \%$ of nurses). Similar results were obtained for case identification. However, less than $70 \%$ of the participants were aware of the mode by which COVID-19 infection was transmitted. In addition, $52.7 \%$ of doctors and $45.3 \%$ of nurses knew about PPE. Interestingly, only $43.2 \%$ of doctors and nurses were aware of proper handwashing and hand hygiene techniques to prevent the transmission of COVID-19.

In terms of preparedness, less than $7 \%$ of both doctors and nurses had taken courses, or training, on COVID-19. About $18 \%$ of participants reported the presence of an isolation room and the availability of a known protocol for isolation. However, only about $13 \%$ of all healthcare workers felt that hospitals were prepared for the COVID-19 outbreak. Meanwhile, $20.6 \%$ of doctors and $26.3 \%$ of nurses perceived themselves as personally prepared for COVID-19. The majority $(65.8 \%)$ also reported that they were not prepared to take

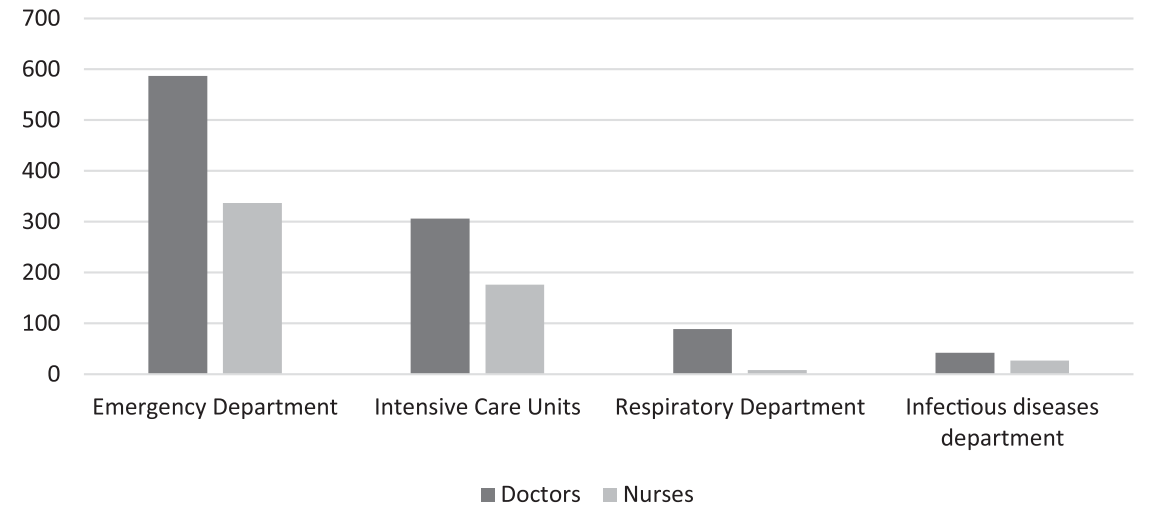

FigURE 1. Distribution of responses by department and profession. 
TABLE 2

Percentage of correct answers on preparedness

\begin{tabular}{|c|c|c|c|}
\hline \multirow[b]{2}{*}{ Question type } & \multicolumn{3}{|c|}{ Answered correctly, $n(\%)$} \\
\hline & Overall & Doctors & Nurses \\
\hline \multicolumn{4}{|l|}{ Knowledge and awareness } \\
\hline $\begin{array}{l}\text { Q1: What are the symptoms of COVID- } \\
19 \text { infections? }\end{array}$ & 42.4 & 44.4 & 31.7 \\
\hline Q2: How to diagnose COVID-19 & 55.9 & 57.5 & 52.9 \\
\hline Q3: COVID-19 case definition & 41 & 40.3 & 42.3 \\
\hline Q4: Identification of at-risk patients & 59.9 & 61.7 & 56.6 \\
\hline $\begin{array}{l}\text { Q5: How to prevent transmission of } \\
\text { COVID-19 }\end{array}$ & 68.8 & 70.3 & 65.9 \\
\hline $\begin{array}{l}\text { Q6: Knowledge of personal protective } \\
\text { equipment (PPE) }\end{array}$ & 50.1 & 52.7 & 45.3 \\
\hline $\begin{array}{l}\text { Q7: Handwashing and type of } \\
\text { Disinfectant }\end{array}$ & 43.2 & 44.2 & 33.3 \\
\hline \multicolumn{4}{|l|}{ Preparedness } \\
\hline $\begin{array}{l}\text { Q1: Have you participated in a training } \\
\text { course for outbreak management? }\end{array}$ & 6.3 & 6.7 & 5.5 \\
\hline $\begin{array}{l}\text { Q2: Protocol for triage and isolation of } \\
\text { suspected cases? }\end{array}$ & 25.2 & 23.8 & 38.4 \\
\hline Q3: Availability of isolation room? & 18.2 & 16.1 & 22.1 \\
\hline $\begin{array}{l}\text { Q4: Are you prepared to manage the } \\
\text { COVID-19 outbreak? }\end{array}$ & 22.6 & 20.6 & 26.3 \\
\hline $\begin{array}{l}\text { Q5: Do you consider your hospital to be } \\
\text { prepared for the COVID-19 } \\
\text { outbreak? }\end{array}$ & 13.4 & 13.4 & 13.5 \\
\hline $\begin{array}{l}\text { Q6: Are you prepared to properly use } \\
\text { PPE? }\end{array}$ & 54.3 & 54 & 54.9 \\
\hline $\begin{array}{l}\text { Q7: Do you know the isolation } \\
\text { procedure? }\end{array}$ & 36.1 & 35.6 & 37 \\
\hline $\begin{array}{l}\text { Q8: Do you know how to report a } \\
\text { potential COVID- } 19 \text { case? }\end{array}$ & 26.3 & 25 & 28.8 \\
\hline $\begin{array}{l}\text { Q9: Do you know what to do if you have } \\
\text { signs of the COVID-19 infection? }\end{array}$ & 42.3 & 45.1 & 37 \\
\hline $\begin{array}{l}\text { Q10: Do you know the safety } \\
\text { precautions that should be taken for } \\
\text { aerosol transmission in patients with } \\
\text { COVID-19? }\end{array}$ & 34.2 & 35.1 & 32.5 \\
\hline $\begin{array}{l}\text { Q11: Do you know the criteria to guide } \\
\text { the evaluation of persons under } \\
\text { investigation? }\end{array}$ & 22.1 & 21.2 & 23.9 \\
\hline
\end{tabular}

precautions to prevent aerosol transmission via COVID-19 patients.

The majority $(73.5 \%)$ reported an inadequate level of knowledge on COVID-19. There was no significant association between hospital department type and level of participant preparedness $(P=0.319)$ or knowledge $(P=0.166)$. Tables 3 and 4 show the level of preparedness and knowledge among healthcare workers by department.

\section{DISCUSSION}

A significant number of healthcare workers expressed low levels of awareness and preparedness regarding COVID-19. This raises a concern regarding the ability of the Libyan healthcare system and its healthcare workers to combat COVID-19 infection. Despite these concerns, along with the poor local healthcare infrastructure in Libya, healthcare workers continue to work during COVID-19, risking their lives to save their patients. Meanwhile, no official courses or training programs are available, and healthcare workers have to purchase PPE themselves, as they are not provided by the hospitals in adequate amounts. Our study provides considerable insights into the necessity of immediate and determined efforts focused on training programs and providing an adequate supply of PPE to alleviate these challenges during the COVID-19 pandemic.

Inadequate knowledge is a risk factor for disease transmission, as it can lead to low levels of care. Our study demonstrated that only $6.7 \%$ of doctors and $5.5 \%$ of nurses had participated in training courses. Our study also reported that about $70 \%$ of participants received information from social media, which is lower than previously reported (91.1\%). Furthermore, our study indicated the lowest rate of knowledge compared with previous studies, where we found that the overall rate of respondents providing correct answers on the knowledge questionnaire was a mere $26.5 \%$, compared with a previous study reporting that $90 \%$ of healthcare workers provided correct answers. ${ }^{23}$ Another recent study demonstrated that $89 \%$ of healthcare workers had sufficient knowledge on COVID-19. ${ }^{24}$

Another primary concern emerging from this study is that only $21.2 \%$ of doctors and $23.9 \%$ of nurses knew the criteria for evaluating persons under investigation for COVID-19 infection. In addition, only about $25 \%$ of doctors knew how to report potential COVID-19 cases, which could prompt an unexpected increase in undiagnosed cases, thereby increasing the burden of infection within the community. Moreover, approximately $18 \%$ of participants were unaware of isolation room specifications and processes for potential COVID-19 patients, which could increase the risk of infection within hospitals.

Interestingly, only $44.2 \%$ of doctors and $33.3 \%$ of nurses were aware of the proper handwashing and hand hygiene techniques and disinfectants. In addition, about $45.1 \%$ of doctors and $37 \%$ of nurses were not prepared to manage a case with signs and symptoms of COVID-19 infection. This could highlight the risk of cross-contamination within hospitals and could lead to a higher rate of hospital-acquired infections. Training and safety precautions, focused on the direct decontamination of contact points among healthcare workers, are needed to prevent the spread of infection. ${ }^{25,26}$

The majority of healthcare workers (77.4\%) felt personally unprepared to address COVID-19 infection. A total of $50.1 \%$ of participants were uneducated about PPE, whereas about $54.3 \%$ were not trained to use it. In addition, PPE is very limited, and hospital workers reported that they independently purchase their own PPE because of the inadequate supply provided by Libyan hospitals. Furthermore, about $35.1 \%$ of

TABLE 3

Participant scores regarding preparedness $(n=1,572)$

\begin{tabular}{lrr}
\hline \multicolumn{1}{c}{ Department } & Adequate $(\geq 8), n(\%)$ & Inadequate $(<8), n(\%)$ \\
\hline Overall & $123(7.8)$ & $1,448(92.2)$ \\
Emergency department & $68(7.4)$ & $855(92.6)$ \\
Intensive care units & $39(8.1)$ & $443(91.9)$ \\
Respiratory department & $8(6.9)$ & $108(93.1)$ \\
Infectious disease department & $8(16)$ & $42(84)$ \\
\hline
\end{tabular}


TABLE 4

Participant scores regarding knowledge and awareness $(n=1,572)$

\begin{tabular}{|c|c|c|c|}
\hline Department & High knowledge $(\geq 5) n(\%)$ & Low knowledge $(<5), n(\%)$ & $x^{2}$ \\
\hline Overall & $416(26.5)$ & $1,156(73.5)$ & 0.319 \\
\hline Emergency department & $241(26.1)$ & 683 (73.9) & \\
\hline Intensive care units & $131(27.2)$ & $351(72.8)$ & \\
\hline Respiratory department & $26(22.4)$ & 90 (77.6) & \\
\hline Infectious disease department & $18(36)$ & 32 (64) & \\
\hline
\end{tabular}

doctors and $32.5 \%$ of nurses were unprepared to take safety precautions to prevent aerosol transmission via individuals with suspected COVID-19 infection. Only about $68 \%$ of the participants were aware of measures to prevent transmission of the COVID-19 virus. These issues raise fear and concerns for safety of healthcare workers and their ability to access PPE safety measures; this calls into question the healthcare system's ability to prevent hospital-acquired infection. Hospitals can also become sites of contamination that spread COVID19 infections into the community; furthermore, healthcare workers can also pass on the infection to family members at home. ${ }^{27}$

Our study found that doctors and nurses were buying PPE themselves, prompting questions around the hospitals' inabilities to provide this essential equipment. Only a small proportion (13.4\%) of participants perceived their hospital as prepared for the outbreak. More training and education are needed on the triage and isolation of suspected cases, as approximately $25 \%$ of participants are not prepared, or trained, to conduct these protocols.

Limitations. Although the study provided a large-scale sample from 21 healthcare centers, and used a sample size that was sufficiently large enough to allow the adequate assessment of healthcare workers' knowledge and preparedness regarding COVID-19 infection, some limitations should still be clarified. The study was conducted in a single country with low resource levels and a lower number of detected COVID-19 infections than other countries, which may have affected the results. Future multinational studies, using more extensive and varied populations, are needed to validate these findings.

\section{CONCLUSION}

In conclusion, our study has illuminated the current level of knowledge and awareness of COVID-19 among doctors and nurses, with special consideration for those working in departments responsible for caring for COVID-19 patients. We focused on healthcare workers who may come into direct contact with COVID-19 patients, and are thus expected to have adequate knowledge and preparedness. By contrast, other studies have focused on more general populations of healthcare workers. ${ }^{23,24,28,29}$ This study provides an overview of healthcare workers' preparedness regarding the current pandemic. The respondents had a lower level of preparedness, which highlights the importance of education and training programs for healthcare workers, to control and prevent infection from COVID-19. However, the absence of an organized and effective governmental plan, along with a poor healthcare infrastructure, renders developing countries vulnerable. Moreover, educational initiatives, along with more tangible forms of support, such as the provision of PPE, should be carried out to help developing countries improve their abilities to control and prevent COVID-19 infection.
Received April 21, 2020. Accepted for publication June 14, 2020.

Published online June 18, 2020.

Note: Supplemental materials are available from the authors upon request.

Acknowledgments: We would like to thank all of the staff who voluntarily participated in this study. Publication charges for this article were waived due to the ongoing pandemic of COVID-19.

Authors' addresses: Muhammed Elhadi, Ahmed Msherghi, Mohammed Alkeelani, Abdulaziz Zorgani, Ahmed Zaid, Ali Alsuyihili, Anis Buzreg, Hazim Ahmed, Ahmed Elhadi, Ala Khaled, Samer Khel, Rawanda Gaffaz, Ayiman Alhashimi, Marwa Biala, Siraj Abulmida, Mohamed Abukhashem, Esra Alghanai, Taha Khaled, Nafati Alnafati, Moaz Alwarfalli, Amna Elmabrouk, Sarah Alhaddad, Farah Madi, Malack Madi, Ayman AL-Agile, Jalal Ahmid, Ola Elmabrouk, Fatimah Bin Alshiteewi, Hind Alameen, Hala Bikhayr, Tahani Aleiyan, Bushray Almiqlash, Malak Subhi, Mawada Fadel, Abraar Abdullah, Abdulrahman Atewa, and Ala Amshai, Faculty of Medicine, University of Tripoli, Tripoli, Libya, E-mails: muhammed.elhadi.uot@gmail.com, ahmedmsherghi@gmail.com, albshrimohamed@gmail.com,zorgania@ yahoo.com, a.zaid@uot.edu.ly, al4light@gmail.com, anisbuzreg94@ gmail.com, hazemahmed.uot@yahoo.com, ahmed.elhadik1994@ gmail.com, ala.khaled.el@gmail.com, samer17ly@gmail.com, drrgaffaz@ hotmail.com, emanalghrari@yahoo.com, marwabiala07@gmail.com, sirajsalehabulmida@gmail.com, bukhshe@gmail.com, esraaer2@ gmail.com, dr.taha.khaled@gmail.com, nafatyshgra@gmail.com, moezmoez9494@gmail.com, amna.elmabruke@gmail.com, sarahalhaddad6@ gmail.com, frhmadi620@gmail.com, madimalack@gmail.com, ayman.alagile@ gmail.com, jeloo.7888@gmail.com, ola.a.mabrok@gmail.com, fatima. alshtiwie@gmail.com, hind.alamein1995@gmail.com, hlabe240@ gmail.comtahanimokhtar50@gmail.com, eledia092@gmail.com, malaktaha199827@gmail.com, mawadaa.nasser126@gmail.com, abramjib@ gmail.com, abdulrahamatewa@gmail.com, and aloamshaya@gmail.com. Tariq Boughididah, Moutaz Elgzairi, Fatima Elkhfeefi, Mohamed Ismaeil, Belal Faraag, and Majdi Badi, Faculty of Medicine, University of Benghazi, Benghazi, Libya, E-mails: tariq.bugadeda@gmail.com, moutazashour@ yahoo.com, fatma.mousa91@gmail.com, mohamed.alfssi@gmail.com, belalade194@gmail.com, and majdibadi3202@gmail.com. Mohammed Abdelkabir, Faculty of Medicine, Sebha University, Sebha, Libya, E-mail: mohammed.abdelkabir@gmail.com. Sumayyah Bahroun and Abdulmueti Alhadi, Faculty of Medicine, University of Zawia, Az-Zawiyah, Libya, E-mails: sumayyah.ghyth@gmail.com and moate.alhadi@gmail.com. Abdelmunam Elharb, Najah Ben Saleim, Mohamed Eisay, Hana Yahya, and Safeya Alkot, Faculty of Medicine, University of AL-Mergib, Al Khums, Libya, E-mails: abdelmoneem_93@yahoo.com, najahas352@gmail.com, alfajralfaj@gmail.com, hanna7yahia@gmail.com, and sofya00ali@ gmail.com. Esra Boushi, Faculty of Medicine, Al-Zintan University, Az-Zintan, Libya, E-mail: boshiesraa@gmail.com. Hamad Mughrabi, Faculty of Medicine, Misurata University, Misurata, Libya, E-mail: hamoghrebe22@gmail.com.

This is an open-access article distributed under the terms of the Creative Commons Attribution (CC-BY) License, which permits unrestricted use, distribution, and reproduction in any medium, provided the original author and source are credited.

\section{REFERENCES}

1. WHO, 2020. WHO Director-General's Opening Remarks at the Mission Briefing on COVID-19 - 9 April 2020. Geneva, Switzerland: World Health Organization. Available at: https://www.who.int/dg/ 
speeches/detail/who-director-general-s-opening-remarksat-the-mission-briefing-on-covid-19---9-april-2020. Published online April 18, 2020.

2. WHO, 2020. WHO Director-General's Opening Remarks at the Media Briefing on COVID-19 - 11 March 2020. Geneva, Switzerland: World Health Organization. Available at: https:// www.who.int/dg/speeches/detail/who-director-general-sopening-remarks-at-the-media-briefing-on-covid-19---11-march2020. Published online April 18, 2020.

3. WHO, 2020. Director-General's Remarks at the Media Briefing on 2019-nCoV on 11 February 2020. Geneva, Switzerland: World Health Organization.

4. Chan JF et al., 2020. A familial cluster of pneumonia associated with the 2019 novel coronavirus indicating person-to-person transmission: a study of a family cluster. Lancet 395: 514-523.

5. Wang D et al., 2020. Clinical characteristics of 138 hospitalized patients with 2019 novel coronavirus-infected pneumonia in Wuhan, China. JAMA 323: 1061-1069.

6. Chen $\mathrm{N}$ et al., 2020. Epidemiological and clinical characteristics of 99 cases of 2019 novel coronavirus pneumonia in Wuhan, China: a descriptive study. Lancet 395: 507-513.

7. Bajema KL et al., 2020. Persons evaluated for 2019 novel coronavirus - United States, January 2020. MMWR Morb Mortal Wkly Rep 69: 166-170.

8. Zhou F et al., 2020. Clinical course and risk factors for mortality of adult inpatients with COVID-19 in Wuhan, China: a retrospective cohort study. Lancet 395: 1054-1062.

9. Wu Z, McGoogan JM, 2020. Characteristics of and important lessons from the coronavirus disease 2019 (COVID-19) outbreak in China: summary of a report of 72,314 cases from the Chinese center for disease control and prevention. JAMA 323: 1239-1242.

10. Huang $C$ et al., 2020. Clinical features of patients infected with 2019 novel coronavirus in Wuhan, China. Lancet 395: 497-506.

11. CDC COVID-19 Response Team, 2020. Severe outcomes among patients with coronavirus disease 2019 (COVID-19) - United States, February 12-March 16, 2020. MMWR Morb Mortal Wkly Rep 69: 343-346.

12. Cascella M, Rajnik M, Cuomo A, Dulebohn SC, Di Napoli R, 2020. Features, Evaluation and Treatment Coronavirus (COVID-19). Treasure Island, FL: StatPearls Publishing StatPearls Publishing LLC.

13. Wu C et al., 2020. Risk factors associated with acute respiratory distress syndrome and death in patients with coronavirus disease 2019 pneumonia in Wuhan, China. JAMA Intern Med Published online March 13, 2020. https://doi.org/10.1001/ jamainternmed.2020.0994.

14. Munoz-Price LS et al., 2019. Infection prevention in the operating room anesthesia work area. Infect Control Hosp Epidemiol 40: $1-17$.

15. Khan Z, Karatas Y, 2020. COVID-19 in Turkey: an urgent need for the implementation of preparedness and response strategies. Health Sci Rep 3: e153.
16. WHO, 2020. Critical Preparedness, Readiness and Response Actions for COVID-19. Geneva, Switzerland: World Health Organization. Available at: https://www.who.int/docs/default-source/ coronaviruse/20200307-cccc-guidance-table-covid19-final.pdf? sfvrsn=1c8ee193_10. Published online April 18, 2020.

17. Libya NCfDC, 2020. COVID-19 Status in April 10, 2020. Available at: https://covid19.ly/. Published online April 18, 2020.

18. WHO, 2017. Health Emergencies and Humanitarian Response Update. Geneva, Switzerland: World Health Organization. Available at: https://www.who.int/hac/crises/lby/sitreps/en/. Published online April 18, 2020.

19. Ughasoro MD, Esangbedo DO, Udorah IM, 2019. Health-care workers' perspectives on preparedness of health-care facilities for outbreak of communicable diseases in Nigeria: a qualitative study. Am J Trop Med Hyg 100: 1022-1028.

20. Khan MU, Shah S, Ahmad A, Fatokun O, 2014. Knowledge and attitude of healthcare workers about middle east respiratory syndrome in multispecialty hospitals of Qassim, Saudi Arabia. BMC Public Health 14: 1281.

21. Kim JS, Choi JS, 2016. Middle east respiratory syndrome-related knowledge, preventive behaviours and risk perception among nursing students during outbreak. J Clin Nurs 25: 2542-2549.

22. Ughasoro MD, Esangbedo DO, Udorah IM, 2019. Health-care workers' perspectives on preparedness of health-care facilities for outbreak of communicable diseases in Nigeria: a qualitative study. Am J Trop Med Hyg 100: 1022-1028.

23. Zhong B-L, Luo W, Li H-M, Zhang Q-Q, Liu X-G, Li W-T, LiY, 2020. Knowledge, attitudes, and practices towards COVID-19 among Chinese residents during the rapid rise period of the COVID-19 outbreak: a quick online cross-sectional survey. Int J Biol Sci 16: 1745-1752.

24. Zhang M, Zhou M, Tang F, Wang Y, Nie H, Zhang L, You G, 2020. Knowledge, attitude and practice regarding COVID-19 among healthcare workers in Henan, China. J Hosp Infect 105: 183-187.

25. Huh S, 2020. How to train the health personnel for protecting themselves from novel coronavirus (COVID-19) infection during their patient or suspected case care. J Educ Eval Health Prof 17: 10.

26. Shi Y, Wang J, Yang Y, Wang Z, Wang G, Hashimoto K, Zhang K, Liu H, 2020. Knowledge and attitudes of medical staff in Chinese psychiatric hospitals regarding COVID-19. Brain Behav Immun Health 4: 100064.

27. The Lancet, 2020. COVID-19: protecting health-care workers. Lancet 395: 922.

28. Giao H, Thi N, Thi Ngoc Han N, Khanh T, Ngan V, Tam V, Le An P, 2020. Knowledge and attitude toward COVID-19 among healthcare workers at district 2 hospital, Ho Chi Minh city. Asian Pac J Trop Med 13: 260-265.

29. Khan S, Khan M, Maqsood K, Hussain T, Noor UI H, Zeeshan M, 2020. Is Pakistan prepared for the COVID-19 epidemic? A questionnaire-based survey. J Med Virol 92: 824-832. 